\title{
Controlling Stiction in Nano-Electro-Mechanical Systems Using Liquid Crystals
}

\author{
Oleksandr Buchnev ${ }^{1}$, Nina Podoliak ${ }^{1}$, Thomas Frank ${ }^{1,2}$, Malgosia Kaczmarek ${ }^{2}$, Liudi Jiang ${ }^{3}$ and \\ Vassili A. Fedotov ${ }^{1, *}$ \\ ${ }^{1}$ Optoelectronics Research Centre, University of Southampton, SO17 1BJ, UK \\ ${ }^{2}$ Physics and Astronomy, University of Southampton, SO17 1BJ, UK \\ ${ }^{3}$ Faculty of Engineering and the Environment, University of Southampton, SO17 1BJ, UK \\ *e-mail: vaf@orc.soton.ac.uk
}

\begin{abstract}
Stiction is one of the major reliability issues limiting practical application of nano-electro-mechanical systems (NEMS), an emerging device technology that exploits mechanical movements on the scale of an integrated electronic circuit. We report on a discovery that stiction can be eliminated by infiltrating NEMS with nematic liquid crystals. We demonstrate this experimentally using a NEMS-based tuneable photonic metamaterial, where reliable switching of optical response was achieved for the entire range of nano-scopic structural displacements admitted by the metamaterial design. Being a more straightforward and easy-to-implement alternative to the existing anti-stiction solutions, our approach also introduces an active mechanism of stiction control, which enables toggling between stiction-free and the usual (stiction-limited) regimes of NEMS operation. It is expected to greatly expand the functionality of electro-mechanical devices and enable the development of adaptive and smart nano-systems.
\end{abstract}

Keywords: stiction; liquid crystals; re-configurable metamaterials; NEMS; MEMS

Nano-electro-mechanical systems (NEMS) are integrated miniature devices that combine electrical and mechanical components at the nano-scale. They have demonstrated great potential for a wide range of applications including nano-actuators and sensors ${ }^{1-3}$ electromagnetic detectors and quantum transducers, ${ }^{4-6}$ low power electronic/optical switches and relays ${ }^{7-9}$ memory ${ }^{10-12}$ and, recently, re-configurable metamaterials and plasmonic devices. ${ }^{13-16}$ The advancement of NEMS technology, however, is hampered by reliability issues such as 'stiction' - unintentional adhesion between compliant surfaces of the mechanical components, which leads to their permanent damage. ${ }^{17,18}$

Stiction occurs when surface adhesion forces such as capillary, van der Waals or electrostatic forces (as well as their combinations) are stronger than the mechanical restoring force exhibited by an actuator. Although stiction can normally be prevented at the device fabrication stage by changing the surface hydrophobicity, or by eliminating liquid-vapor interfaces through supercritical fluid, freeze-drying or dry-release methods, ${ }^{19-21}$ failure may still arise due to changes in ambient conditions, or during routine operation of the device when its mechanical components come into close contact with each other. Currently employed methods of tackling stiction include surface treatment and coatings, ${ }^{22-24}$ and tailoring the designs of cantilevers and contact electrodes. ${ }^{25,26}$ Being recognized as the major problem in micro-scale electromechanical systems

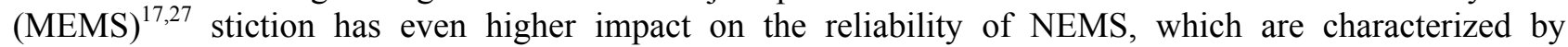
substantially larger surface-to-volume ratio. In the latter case, however, the application of stiction-preventive measures that have been found effective for MEMS $S^{23,28-30}$ is quite challenging given the scale of NEMS devices.

Here we show that infiltrating NEMS with nematic liquid crystals (as illustrated in Figure 1a) yields a simple, yet efficient, alternative to the existing anti-stiction solutions, and enables robust control of nanoscopic actuations in NEMS for the entire range of physically allowed displacements. Our approach also enables on-demand, fully reversible transition between stiction-free and stiction-limited regimes of NEMS operation, which could greatly expand the functionality of the integrated electro-mechanical systems.

\section{RESULTS AND DISCUSSION}

In the reported study NEMS was represented by a re-configurable planar metamaterial (metasurface) - a nano-patterned thin metal film with diffraction-free resonant response that can be tuned mechanically, via electrically induced displacements in the fabric of the structure. ${ }^{31}$ Our choice of this particular type of NEMS was determined by the convenience of detecting nano-scopic structural displacements, which for a metamaterial can be done optically, by observing modifications of its transmission spectra. Our metamaterial 
was based on a periodic array of connected V-shaped metallic nano-resonators, a tried-and-tested design ${ }^{32-34}$ that resembles a continuous 'zig-zag' wire grid (see Figures 1a and 1b). The grid (gold) was attached to, and fully rested on, an array of dielectric nano-bridges (silicon nitride). The nano-bridges had the same shape and width as the wires of the grid (Figure 1c), and could mechanically flex in the plane of the array. To reduce the stiffness of the metamaterial structure its zig-zag sections received straight extensions at both ends (see Figure 1b). Adjacent wires were electrically grouped into pairs in an alternating fashion, such that odd and even pairs were disconnected from the opposite sides of the gold frame, as shown in Figure 1d. Those modifications of the design allowed us to apply a potential difference between the pairs, and induce uniform lateral displacements of the zig-zag sections via the combination of electrostatic attraction and repulsion between the wires, as illustrated in Figure 1a. Overall, the metamaterial structure was configured to operate in the near-IR, exhibiting plasmonic resonance as a peak in the transmission spectrum at around $0.9 \mu \mathrm{m}$ (see Figure 2d).
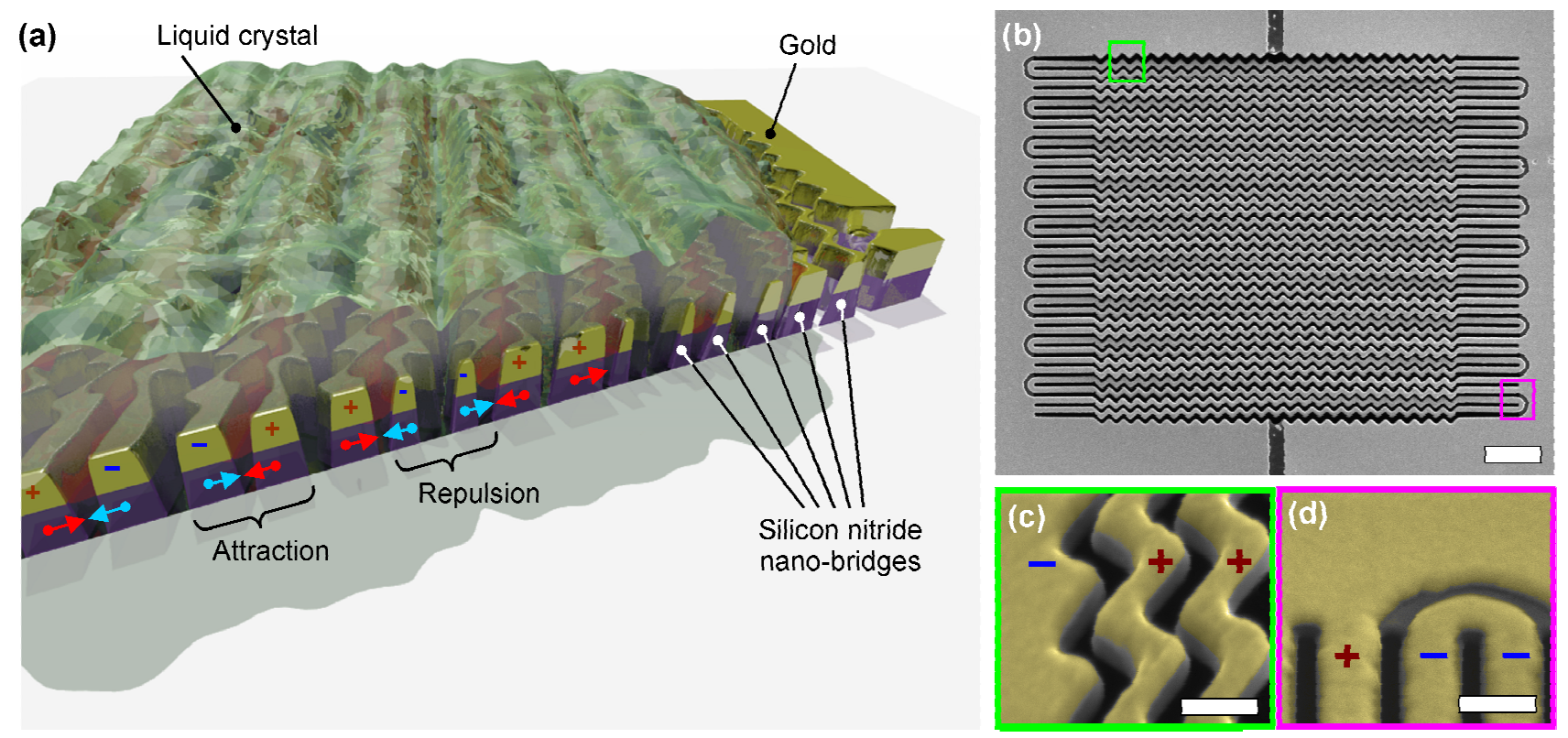

Figure 1. Nano-electro-mechanical system under study. (a) An artistic impression of a mechanically re-configurable zig-zag metasurface (cross-section) infiltrated with a liquid crystal. Red and blue arrows indicate the directions of electrically-induced displacements for the nano-bridges baring opposite potentials. (b) SEM image of the fabricated metasurface. Scale bar corresponds to $2 \mu \mathrm{m}$. (c) \& (d) Close up view of the areas marked in panel (b) with green and purple boxes, respectively. Images were taken with SEM at $52^{\circ}$ to the normal and colour-coded to enhance the contrast between gold (yellow) and silicon nitride (grey). Scale bar corresponds to $300 \mathrm{~nm}$. Symbols '+' and '-' indicate the sign of the electrical potential applied to the corresponding wires.

The failure of the re-configurable metamaterial due to stiction was first observed directly under a scanning electron microscope (Figures 2a-2c, for movies see Supporting Information). The nano-bridges that bore the wires connected to opposite potentials came into physical contact with each other at $V_{\mathrm{p}}=2.7 \mathrm{~V}$ (pull-in voltage) and remained stuck upon withdrawal of the voltage. That permanently transformed the pattern of the metamaterial from a single- to a double-wire zig-zag grid (Figure 2c), which was also detected optically as an irreversible change in the transmission spectrum of a pristine metamaterial sample (see Figures $2 \mathrm{~d}$ and 2e). In particular, with the potential difference ramping from 0 to $2.9 \mathrm{~V}$ for the first time the level of transmission at the resonance was seen to decrease from about $53 \%$ to $48 \%$, falling sharply between 2.3 and $2.9 \mathrm{~V}$. The level of transmission remained unchanged when we increased the bias voltage further, past $2.9 \mathrm{~V}$, and then ramped it down to $0 \mathrm{~V}$ (see Figure 2e).

Similar behaviour was observed for a pristine metamaterial sample infiltrated with a liquid crystal (LC) in the isotropic phase, namely kept above the clearing temperature. The main difference was that the presence of LC shifted the metamaterial resonance by about $0.3 \mu \mathrm{m}$ towards the IR and marginally affected the overall transparency of the sample, which exhibited permanent switching from $43 \%$ to $30 \%$ (see Figures $3 a$ and $3 b$ ). Also, local increase of electric permittivity caused by the presence of LC had affected the pull-in voltage range, which shifted to higher values with the lower and upper bounds being 5.0 and $6.5 \mathrm{~V}\left(V_{\mathrm{p}} \approx 5.8 \mathrm{~V}\right)$. 
Once the potential difference exceeded $6.5 \mathrm{~V}$ the transmission spectrum would not change, regardless of the bias applied thereafter, signifying mechanical damage of the re-configurable structure.

The situation changed dramatically when we cooled the apparently damaged, LC-infiltrated sample to room temperature, thus forcing the transition of LC from the isotropic to the nematic phase. Firstly, upon lowering the temperature at $0 \mathrm{~V}$, the transmission spectrum seemed to recover to its initial state - as had been observed just after the infiltration of the metamaterial (Figure 3c). A minor change is attributed to the optical anisotropy of LC, which in our case was relatively week and expected to result in a spectral shift of less than 3\% (see Supporting Information). Next, we regained the ability to tune the resonant response of the metamaterial within the previously established limits of the transmission level (43-30\%) and bias voltage $(0-6.5 \mathrm{~V})$, although this time in a continuous and reversible manner. In particular, when we ramped the voltage up, the level of transmission decreased more gradually, without a sharp drop characteristic to the onset of stiction (see Figure 3d). The change of the metamaterial response was complete at $6.5 \mathrm{~V}$ with no further modifications of the spectrum visible at higher voltage (increasing the voltage past $7 \mathrm{~V}$ often led to electrical breakdown of the samples). However, contrary to the previous scenarios (involving isotropic LC or no LC), the transparency of the sample was also seen to recover during the voltage ramp-down, as soon as the potential difference dropped below $6.5 \mathrm{~V}$. Figure $3 \mathrm{~d}$ shows that the trends of the transmission change measured with increasing and decreasing bias voltage overlapped except for the very first ramp-up-rampdown cycle, when a slight hysteresis was observed.
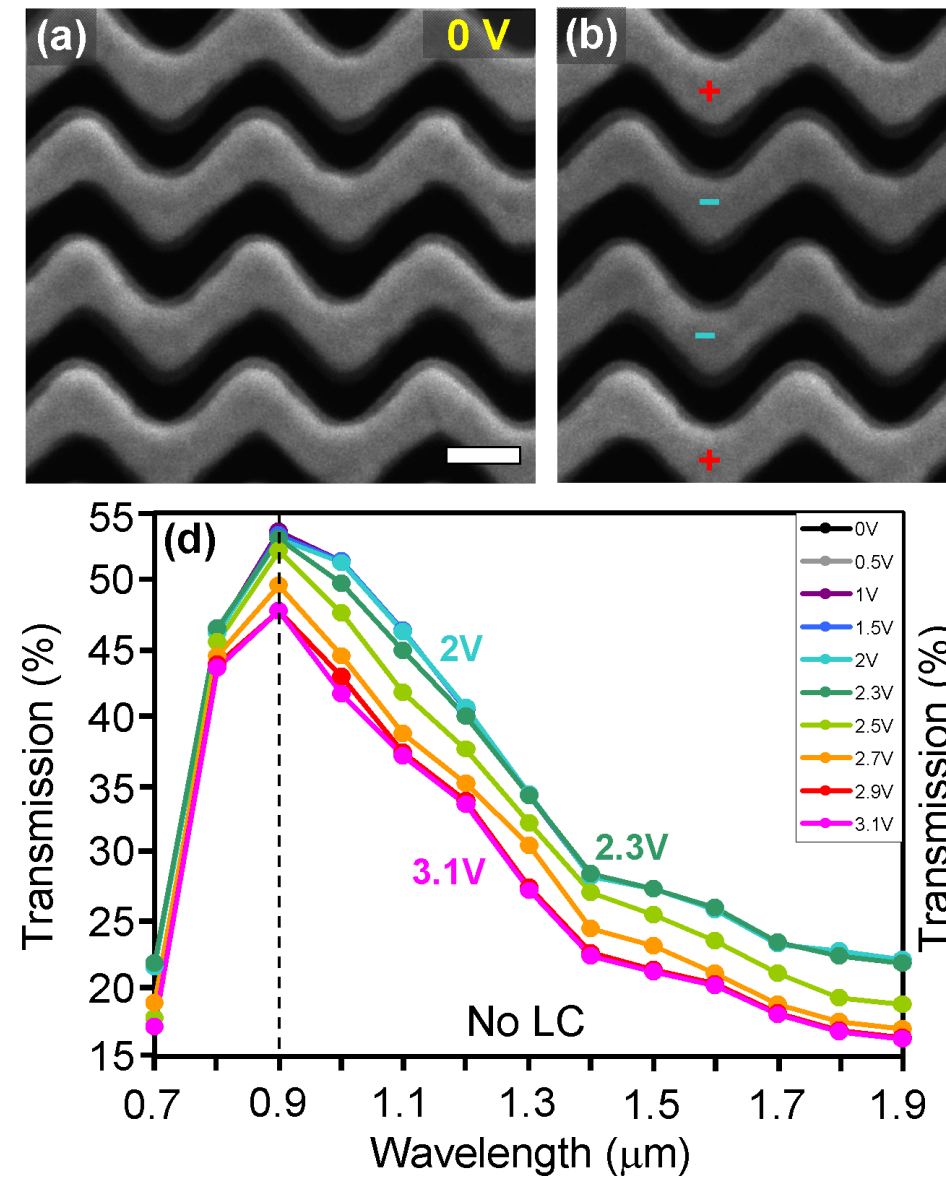
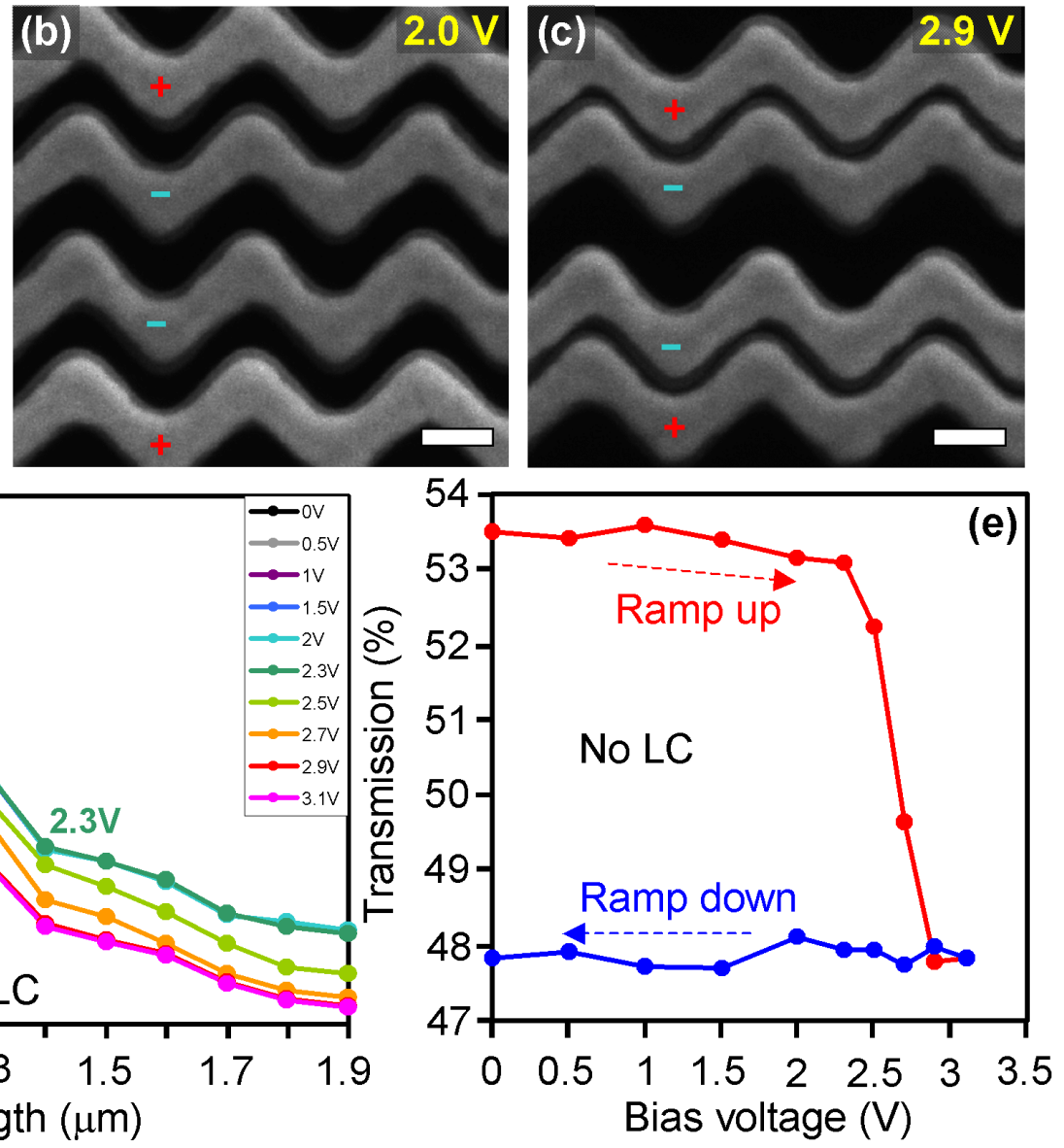

Figure 2. Irreversible electro-mechanical switching of re-configurable metasurface. (a) - (c) SEM images of a fragment of the metamaterial wire grid captured at $0 \mathrm{~V}$ (a), $2.0 \mathrm{~V}$ (b) and $2.7 \mathrm{~V} \mathrm{(c)} \mathrm{in} \mathrm{the} \mathrm{course} \mathrm{of} \mathrm{electro-mechanical}$ switching. Scale bar corresponds to $200 \mathrm{~nm}$. Symbols '+' and '-' indicate the sign of the electrical potential applied to the corresponding wires. (d) Transmission spectrum of the metasurface acquired at different bias voltage during the first voltage ramp up in the range $0-3.1 \mathrm{~V}$. Dashed line marks the position of the metamaterial resonance at $0 \mathrm{~V}$. (d) Transmission of the metasurface measured at the resonance wavelength $(0.9 \mu \mathrm{m})$ while ramping bias voltage up and down for the first time. 
Remarkably, by cycling the phase of LC between nematic and isotropic we were able to toggle between reversible (Figure 3b) and irreversible (Figure 3d) regimes of controlling metamaterial transmission.

While irreversible switching of an LC-infiltrated sample was a clear manifestation of NEMS stiction, the discovered regime of its reversible operation must have also engaged actuations of the nano-bridges, yet somewhat immune to stiction. Indeed, according to our simulations (see Supporting Information), the reorientation of nematic director in the bulk of LC layer and the corresponding changes of optical anisotropy would have occurred below $3.0 \mathrm{~V}$. Also, the measured overall change of the metamaterial transmission in the presence of nematic and isotropic LC had the same magnitude, which together with the simulation results rules out electrically controlled birefringence as the only mechanism for the observed transmission variation. Besides, toggling between reversible and irreversible regimes of metamaterial optical switching could only be possible if the nano-bridges became unstuck upon cooling the sample and were free to move while LC remained in the nematic phase. The behaviour of metamaterial transmission around $6.5 \mathrm{~V}$ (Figures $3 \mathrm{~b} \& 3 \mathrm{~d}$ ) suggests that the magnitude of the structural deformation at the end of the voltage ramp-up reached the physically allowed limit, where every other gap between the nano-bridges had closed up. That brought the nano-bridges within the effective range of the surface adhesion forces and should have resulted in stiction, just as it occurred while LC was in the isotropic phase. Below we discuss possible mechanisms of the stiction suppression, deferring an in-depth quantitative analysis of the effect to a future publication.
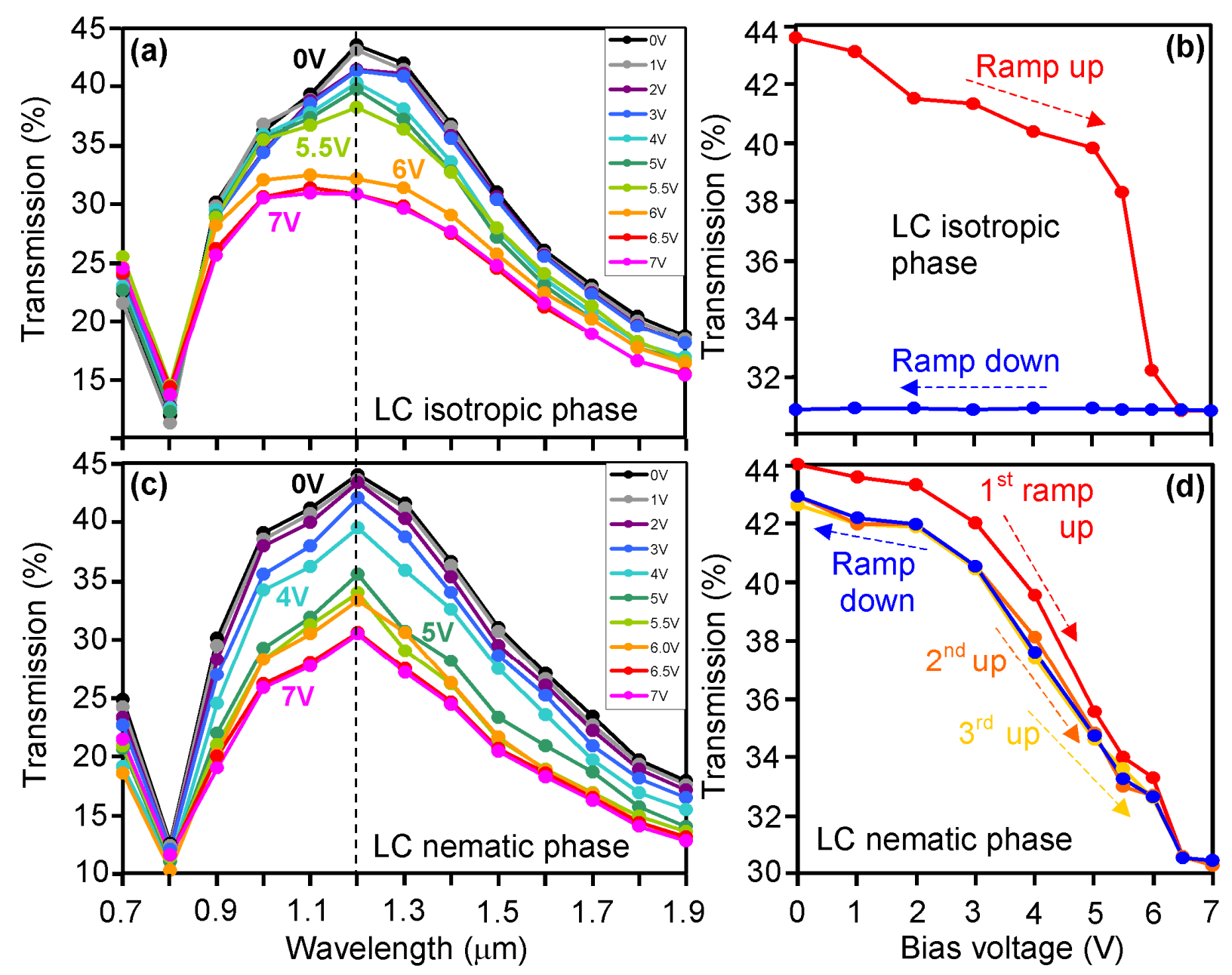

Figure 3. Switching behaviour of re-configurable metasurface infiltrated with liquid crystal. (a) \& (c) Transmission spectrum of the metasurface measured at different bias voltage during the first voltage ramp up in the range $0-7 \mathrm{~V}$ while maintaining the liquid crystal in isotropic (a) and nematic (c) states, respectively. Dashed lines mark the position of the metamaterial resonance at $0 \mathrm{~V}$. (b) \& (d) Transmission of the metasurface measured for correspondingly isotropic and nematic states of the liquid crystal at the resonance wavelength $(1.2 \mu \mathrm{m})$ while ramping bias voltage up and down. 
The main failure mode of electrostatically driven actuators and capacitive switches that is related to stiction is dielectric charging. ${ }^{35,36}$ The reduction of parasitic, electrostatic attraction between silicon nitride nanobridges (which are charged to at least $6.5 \mathrm{~V}$ upon irreversible switching) is therefore one of the likely causes of the observed stiction-free behaviour. In particular, one may expect the attraction force $F_{\text {att }}$ to decrease as the result of a change of the local dielectric permittivity $\varepsilon$ during the transition of LC to the nematic state. In this case the permittivity of LC becomes highly anisotropic, while the residual electric field between the nano-bridges is strong enough to deform the LC director locally and orient most of the LC molecules along the field lines. ${ }^{37,38}$ The switching of LC phase from isotropic to nematic is thus accompanied by an increase of the static dielectric constant in the vicinity of the closed gaps and, given that $F_{\text {att }} \propto 1 / \varepsilon$ for electrically isolated charged bodies, can in principle trigger the release of the nano-bridges at zero bias. However, this mechanism of stiction suppression no longer applies during the actuation of the nano-bridges, when the metamaterial wire grid is connected to a voltage source. In this case $F_{\text {att }} \propto \varepsilon$, so at the end of voltage ramp-up the electrostatic attraction mediated by the nematic phase of LC turns out to be even stronger than in the case of isotropic LC (which is also supported by the results of our simulations, presented in the Supporting Information). It cannot be counteracted by the London dispersion forces, since for a symmetric configuration (such as a pair of silicon nitride boundaries separated by a dielectric layer) they were shown to be always attractive ${ }^{39,40}$ We also rule out electric discharge as an anti-stiction mechanism specific to nematic phase - it would involve localised heating and hence resulted in distortions of LC, which could be easily observed under polarized optical microscope in the course of our measurements; besides, ion mobility in nematic phase is generally lower than in the isotropic one. ${ }^{41}$

We therefore conclude that stiction-free switching of the re-configurable metamaterial resulted from an increase of the mechanical restoring force, which must have acquired a contribution from elastic distortions of LC. For example, the electric field between closing nano-bridges $(>10 \mathrm{kV} / \mathrm{mm})$ may cause a substantial increase of LC viscosity near the gap ${ }^{42,43}$ and suppress the flow coupling, which will locally render nematic $\mathrm{LC}$ as an elastomer that counteracts the adhesion forces through electrically induced splay and/or bend deformations. Distortions of the nematic director also lead to the appearance of the so-called structural forces. ${ }^{44-46}$ Being the consequence of the long-range orientational order and orientational elasticity of nematic $\mathrm{LC}^{46}$ the structural forces can affect actuations of the nano-bridges within the entire range of physically allowed displacements, and hence may even underpin the more gradual trend of the transmission change observed during the reversible switching (Figure 3d). While stiction-free switching has been demonstrated with $5 \mathrm{CB}$, a standard and well-characterised in the literature nematic liquid crystal, a similar behaviour is expected with other liquid crystals from the same class, such as, for example, E7 and TL205.

\section{CONCLUSIONS}

By infiltrating a NEMS-based re-configurable metasurface with a nematic liquid crystal we were able to prevent permanent adhesion between movable parts of the nanostructure occurring in the course of its mechanical switching. Apart from being a more straightforward and easy-to-implement alternative to the existing anti-stiction solutions, our approach also introduces an active mechanism of stiction control, which enables a fully reversible transition from stiction-free back to the usual (stiction-limited) regime of NEMS operation. The mechanism exploits coupling between mechanical deformations of the nano-actuators and elastic distortions of LC molecular structure arising in ordered LC phases. It is expected to greatly expand the functionality of NEMS/MEMS devices, given high susceptibility of liquid crystals to a wide range of external stimuli including heat, light, electric and magnetic fields. ${ }^{47}$ In particular, controlled stiction could be exploited in non-volatile memory or field-programmable gate arrays based on NEMS. Elasto-mechanical coupling brings together intrinsic non-linearity of liquid crystals and dynamic tuneability of the reconfigurable metamaterials opening a route towards, for example, the realization of magneto-elastic metamaterials $^{48}$ at optical frequencies. Also, a broad spectrum of structural transitions exhibited by liquid crystals at the nano-scale (which includes most notably positional and orientational ordering, ${ }^{49}$ nematic capillary condensation ${ }^{50,51}$ and pre-smectic layering ${ }^{52,53}$ ) should enrich the switching behaviour of LCinfiltrated electro-mechanical actuators and re-configurable meta-devices ${ }^{54}$ near LC clearing point, thus making the latter a practical proposition for developing adaptive and 'smart' nano-systems. 


\section{METHODS}

Sample fabrication: The design of a mechanically re-configurable metasurface was based on a doubleperiodic array of electrically connected V-shaped plasmonic nano-resonators forming a continuous 'zig-zag' wire pattern (see Figure 1b). The metasurface was fabricated by milling, with a focused ion-beam, a $60 \mathrm{~nm}$ thick gold film that had been sputtered beforehand on a $50 \mathrm{~nm}$ thick ultra low-stress silicon nitride membrane (Norcada). The resulting gold wires had the width of approximately $160 \mathrm{~nm}$ and were separated by $100 \mathrm{~nm}$ wide gaps. The unit cell of zig-zag pattern was rectangular with the size of $340 \mathrm{~nm} \times 490 \mathrm{~nm}$, which rendered our planar metamaterial as non-diffracting in the visible and near-IR, at wavelengths larger than $0.5 \mu \mathrm{m}$. The milling also completely removed parts of the membrane in the gaps between the nano-wires, thus yielding a gold zig-zag grid fully suspended on silicon nitride nano-bridges (Figure 1c). The zig-zag sections of the metasurface also received $2 \mu \mathrm{m}$ long straight extensions at both ends, which helped to reduce the stiffness of the metamaterial structure. The nano-wires were grouped into pairs with the odd and even pairs being electrically disconnected from the opposite sections of the remaining gold film (Figure 1d), which had been split to act as macroscopic DC voltage terminals (as illustrated in Figure 1a). The overall size of the re-configurable metasurface was $13 \mu \mathrm{m} \times 17 \mu \mathrm{m}$.

Infiltration with liquid crystal: The metamaterial was infiltrated with a nematic liquid crystal 5CB (Merck). Our choice was dictated merely by the convenience of working with $5 \mathrm{CB}$, which has the lowest clearing temperature among widely available nematic liquid crystals $\left(35^{\circ} \mathrm{C}\right)$, and relatively low viscosity and weak optical anisotropy $\left(n_{\mathrm{o}}=1.520, n_{\mathrm{e}}=1.681, n_{\text {iso }}=1.574\right.$ at $\left.\lambda=1 \mu \mathrm{m}\right) .{ }^{55} 5 \mathrm{CB}$ was applied to the surface of the sample using a drop-casting method (see illustration in Figure 1a). To facilitate optical measurements the drop was squeezed by a glass cover slide yielding a thin, optically uniform LC layer. The glass slide was coated with $20 \mathrm{~nm}$ thick film of unrubbed polyimide, which ensured both parallel alignment and weak anchoring of LC molecules at its surface. The thickness of LC layer was set to $2 \mu \mathrm{m}$ by dielectric spacers placed between the glass cover and gold film. To promote the infiltration we maintained the temperature of the sample above the clearing point of $5 \mathrm{CB}$ for about 10 mins.

Optical measurements: Optical response of the metamaterial sample was characterized in transmission at normal incidence using linearly polarized light. The polarization was set parallel to the nano-wires of zig-zag pattern. Transmission spectra were collected in the range $0.7-1.9 \mu \mathrm{m}$ with a spectral resolution of $0.1 \mu \mathrm{m}$ using a microscope connected to a spectrometer.

\section{ACKNOWLEDGMENTS}

The authors would like to acknowledge the financial support of the UK's Engineering and Physical Sciences Research Council (through grant EP/G00515X/1), and Zepler Institute Stimulus Fund (through grant 515551110). The authors would also like to thank J.-Y. Ou, T. Carr, N. I. Zheludev and K. O'Shea for stimulating discussions and help in preparing the manuscript.

\section{SUPPORTING INFORMATION}

Supporting Information Available: Modelling changes in the optical anisotropy of LC layer and distribution of the control electric field (PDF); includes figures S1, S2 and S3. Movies of a pristine reconfigurable metasurface made under SEM while ramping the control voltage up and down (XviD/AVI). These materials are available free of charge via the Internet at http://pubs.acs.org.

\section{REFERENCES}

1. Ekinci, K. L.; Roukes, M. L. Nanoelectromechanical Systems. Rev. Sci. Instrum. 2005, 76, 061101.

2. Im, H.; Huang, X.-J.: Gu, B.; Choi, Y.-K. A Dielectric-Modulated Field-Effect Transistor for Biosensing. Nat. Nanotechnol.2007, 2, 430-434. 
3. Naik, A. K.; Hanay, M. S.; Hiebert, W. K.; Feng, X. L.; Roukes, X. L. Towards Single-Molecule Nanomechanical Mass Spectrometry. Nat. Nanotechnol. 2009, 4, 445-450.

4. Bagci, T.; Simonsen, A.; Schmid, S.; Villanueva, L. G.; Zeuthen, E.; Appel, J.; Taylor, J. M.; Srensen, A.; Usami, K.; Schliesser, A.; Polzik, E. S. Optical Detection of Radio Waves Through a Nanomechanical Transducer. Nature 2014, 507, 81-85.

5. Akram, U.; Kiesel, N.; Aspelmeyer, M.; Milburn, G. J. Single-Photon Opto-Mechanics in the Strong Coupling Regime. New J. Phys. 2010, 12, 083030.

6. Verhagen, E.; Dele'glise, S.; Weis, S.; Schliesser, A.; Kippenberg, T. J. Quantum-Coherent Coupling of a Mechanical Oscillator to an Optical Cavity Mode. Nature 2012, 482, 63-67.

7. Lee, S. W.; Lee, D. S.; Morjan, R. E.; Jhang, S. H.; Sveningsson, M.; Nerushev, O. A.; Park, Y. W.; Campbell, E. E. B. A Three-Terminal Carbon Nanorelay. Nano. Lett. 2004, 4, 2027-2030.

8. Jang ,W. W.; Lee, J. O.; Yoon, J.-B.; Kim, M.-S.; Lee, J.-M.; Kim, S.-M.; Cho, K.-H.; Kim, D.-W.; Park, D.; Lee. W.-S. Fabrication and Characterization of a Nanoelectromechanical Switch with 15-nm-Thick Suspension Air Gap. Appl. Phys. Lett. 2008, 92,103110.

9. Loh, O. Y.; Espinosa, H. D. Nanoelectromechanical Contact Switches," Nat. Nanotechnol. 2012, 7, 283295.

10. Jang, J. E.; Cha, S. N.; Choi, Y. J.; Kang, D. J.; Butler, T. P.; Hasko, D. G.; Jung, J. E.; Kim, J. M.; Amaratunga, G. A. J. Nanoscale Memory Cell Based on a Nanoelectromechanical Switched Capacitor. Nat. Nanotechnol. 2008, 3, 26-30.

11. Rueckes, T.; Kim, K.; Joselevich, E.; Tseng, G. Y.; Cheung, C.-L.; Lieber, C. M. Carbon NanotubeBased Nonvolatile Random Access Memory for Molecular Computing. Science 2000, 289, 94-97.

12. Lee, S. W.; Park, S. J.; Campbell, E. E.; Park, Y. A Fast and Low-Power Microelectromechanical System-Based Non-Volatile Memory Device. Nat. Commun. 2011, 2, 220.

13. Ou, J. Y.; Plum, E.; Zhang, J.; Zheludev, N. I. An Electromechanically Reconfigurable Plasmonic Metamaterial Operating in the Near-Infrared. Nat. Nanotechnol. 2013, 8, 252-255.

14. Yamaguchi, K.; Fujii, M.; Okamoto, T.; Haraguchi, M. Electrically Driven Plasmon Chip: Active Plasmon Filter. Appl. Phys. Express 2014, 7, 012201.

15. Thijssen, R.; Verhagen, E.; Kippenberg, T. J.; Polman, A. Plasmon Nanomechanical Coupling for Nanoscale Transduction. Nano Lett. 2013, 13, 3293-3297.

16. Liu, X.; Padilla, W. J. Dynamic Manipulation of Infrared Radiation with MEMS Metamaterials. $A d v$. Opt. Mater. 2013, 1, 559-562.

17. Maboudian, R.; Howe, R. T. Critical Review: Adhesion in Surface Micromechanical Structures. J. Vac. Sci. Technol. 1997, B15, 1-20.

18. Yapu, Z. Stiction and Anti-Stiction in MEMS and NEMS. Acta Mech. Solida Sin. (Chin. Ed.) 2003, 19, $1-10$.

19. Guckel, H.; Sniegowski, J. J.; Christenson, T. R.; Raissi, F. The Application of Fine Grained, Tensile Polysilicon Mechanically Resonant Transducers. Sens. Actuators 1990, A21-A23, 346-51.

20. Maboudian, R. Surface Processes in MEMS Technology. Surf. Sci. Rep. 1998, 30, 207-269.

21. Maboudian, R., Ashurst, W.R.; Carraro, C. Tribological challenges in micromechanical systems. Tribol. Lett. 2002, 12, 95-100.

22. Mastrangelo, C. H. Adhesion-Related Failure Mechanisms in Micromechanical Devices. Tribol. Lett. 1997, 3, 223-238.

23. de Boer, M. P.; Mayer, T. M. Tribology of MEMS. MRS Bull. 2001, 26, 302-304.

24. Maboudian, R.; Ashurst, W. R.; Carraro, C. Self-Assembled Monolayers as Anti-Stiction Coatings for MEMS: Characteristics and Recent Developments. Sens. Actuators 2000, 82, 219-223.

25. Mercado, L. L.; Kuo, S.-M.; Lee, T.-Y. T.; Liu, L. Mechanics-Based Solutions to RF MEMS

Switch Stiction Problem. IEEE Trans. Compon. Packag. Technol. 2004, 27, 560-567.

26. Lee, J. O.; Song, Y.-H.; Kim, M.-W.; Kang, M.-H.; Oh, J.-S. Yang, H.-H.; Yoon, J.-B. A Sub-1-Volt Nanoelectromechanical Switching Device. Nat. Nanotechnol. 2013, 8, 36-40.

27. Romig, A. D. Jr.; Dugger, M. T.; McWhorter, P. J. Materials Issues in Microelectromechanical Devices: Science, Engineering, Manufacturability and Reliability. Acta Mater. 2003, 51, 5837-5866.

28. Scharf, T. W.; Prasad, S. V.; Dugger, M. T.; Kotula, P. G.; Goeke, R. S.; Grubbs, R. K. Growth, Structure, and Tribological Behavior of Atomic Layer-Deposited Tungsten Disulphide Solid Lubricant Coatings with Applications to MEMS. Acta Mater. 2006, 54, 4731-4743.

29. Asay, D. B.; Dugger, M. T.; Kim, S. H. In Situ Vapor-Phase Lubrication of MEMS. Tribol. Lett. 2008, $29,67-74$. 
30. Ashurst, W. R.; Carraro, C.; Maboudian, R. Vapor Phase Anti-Stiction Coatings for MEMS. IEEE Trans. Device Mater. Reliab. 2003, 3, 173-178.

31. Zheludev, N. I.; Plum, E. Reconfigurable Nanomechanical Photonic Metamaterials. Nat. Nanotechnol. 2016, 11, 16-22.

32. Buchnev, O.; Ou, J. Y.; Kaczmarek, M.; Zheludev, N. I.; Fedotov, V. A. Electro-Optical Control in a Plasmonic Metamaterial Hybridised with a Liquid-Crystal Cell. Opt. Express 2013, 21, 1633-1638.

33. Buchnev, O.; Podoliak, N.; Kaczmarek, M.; Zheludev, N. I.; Fedotov, V. A. Electrically Controlled Nanostructured Metasurface Loaded with Liquid Crystal: Toward Multifunctional Photonic Switch. Adv. Opt. Mater. 2015, 3, 674-679.

34. Valente, J.; Ou, J. Y.; Plum, E.; Youngs, I. J.; Zheludev, N. I. A Magneto-Electro-Optical Effect in a Plasmonic Nanowire Material. Nat. Commun. 2015, 6, 7021.

35. Wibbeler, J.; Pfeifer, G.; Hietschold, M. Parasitic Charging of Dielectric Surfaces in Capacitive Microelectromechanical Systems (MEMS). Sens. Actuators 1998, A71, 74-80.

36. Zaghloul, U.; Papaioannou, G. J.; Coccetti, F.; Pons, P.; Plana, R. A Systematic Reliability Investigation of the Dielectric Charging Process in Electrostatically Actuated MEMS Based on Kelvin Probe Force Microscopy. J. Micromech. Microeng. 2010, 20, 064016.

37. Self, R. H.; Please, C. P.; Sluckin, T. J. Deformation of Nematic Liquid Crystals in an Electric Field. Eur. J. Appl. Math. 2002, 13, 1-23.

38. Gorkunov, M. V.; Osipov, M. A. Tunability of Wire-Grid Metamaterial Immersed into Nematic Liquid Crystal. J. Appl. Phys. 2008, 103, 036101.

39. French, R. H.; Mullejans, H.; Jones, D. J.; Duscher, G.; Cannon, R. M.; Ruhle, M. Dispersion Forces and Hamaker Constants for Intergranular Film in Silicon Nitride from Spatially Resolved Valence Electron Energy Loss Spectrum Imaging. Acta Mater. 1998, 46, 2271-2287.

40. French, R. H. Origins and Applications of London Dispersion Forces and Hamaker Constants in Ceramics. J. Am. Ceram. Soc. 2000, 83, 2117-2146.

41. Derfela, G.; Lipiński, A. Charge Carrier Mobility Measurements in Nematic Liquid Crystals. Mol. Cryst. Liq. Cryst. 1979, 55, 89-100.

42. Tani, J.; Takagi, T.; Nakaniwa, H.; Ohtomo, K.; Kosugo, K. Vibration Suppression Effect of Liquid Crystal under Electromagnetic Field. J. Intell. Mater. Syst. Struct. 1996, 3, 272-277.

43. Morishita, S.; Nakano, K.; Kimura, Y. Electroviscous Effect of Nematic Liquid Crystals. Tribol. Int. 1993, 26, 399-403.

44. Horn, R. G.; Israelachvili, J. N. Direct Measurement of Structural Forces Between 2 Surfaces in a NonPolar Liquid. J. Chem. Phys. 1981, 75, 1400-1411.

45. Poulin, P.; Stark, H.; Lubensky, T. C.; Weitz, D. A. Novel Colloidal Interactions in Anisotropic Fluids. Science 1997, 275, 1770-1773.

46. Musevic, I. Forces in Nematic Liquid Crystals: from Nanoscale Interfacial Forces to Long-Range Forces in Nematic Colloids. Liq. Cryst. 2009, 36, 639-647.

47. de Gennes, P. G.; Prost, J. The Physics of Liquid Crystals; Oxford University Press, 1995.

48. Lapine, M.; Shadrivov, I. V.; Powell D. A.; Kivshar, Y. S. Magnetoelastic Metamaterials. Nat. Mater. 2012, 11, 30-33.

49. Ruths, M.; Steinberg, S.; Israelachvili, J. N. Effects of Confinement and Shear on the Properties of Thin Films of Thermotropic Liquid Crystal. Langmuir 1996, 12, 6637-6650.

50. Poniewierski, A.; Sluckin, T. J. Theory of the Nematic-Isotropic Transition in a Restricted Geometry. Liq. Cryst. 1987, 2, 281-311.

51. Kocevar, K.; Borstnik, A.; Musevic, I.; Zumer, S. Capillary Condensation of a Nematic Liquid Crystal Observed by Force Spectroscopy. Phys. Rev. Lett. 2001, 86, 5914-5917.

52. de Gennes, P. G. Interactions Between Solid Surfaces in a Presmestic Fluid. Langmuir 1990, 6, 14481450.

53. Ziherl, P. Structural Force in a Presmectic Liquid. Phys. Rev. E 2000, 61, 4636-4639.

54. Zheludev, N. I.; Kivshar, Y. S. From Metamaterials to Metadevices. Nat. Mater. 2012, 11, 917-924.

55. Li, J.; Wen, C.-H.; Gauza, S.; Lu, R.; Wu, S.-T. Refractive Indices of Liquid Crystals for Display Applications. IEEE/OSA J. Disp. Technol. 2005, 1, 51-61. 